Journal of Parenteral and Enteral

\title{
Morphomic Malnutrition Score: A Standardized Screening Tool for Severe Malnutrition in Adults
}

Nutrition

Volume 42 Number 8

November 2018 1263-1271

(c) 2018 American Society for

Parenteral and Enteral Nutrition

DOI: 10.1002/jpen.1175

wileyonlinelibrary.com

WILEY

\author{
Christopher Lee, BS ${ }^{1}$; Erica Raymond, MS, RD, CNSC ${ }^{2}$; Brian A. Derstine, MS ${ }^{1}$ (D); \\ Joshua M. Glazer, $\mathrm{MD}^{\mathbf{3}}$; Rebecca Goulson, $\mathrm{BS}^{\mathbf{1}}$; Avinash Rajasekaran, $\mathrm{BS}^{\mathbf{1 , 4}}$; \\ Jill Cherry-Bukowiec, MD, MS, PNS, FACS ${ }^{5}$; Grace L. Su, MD ${ }^{1,5,6,7}$; \\ and Stewart C. Wang, MD, PhD, FACS ${ }^{1,5}$
}

\begin{abstract}
Background: Granular diagnostic criteria for adult malnutrition are lacking. Objective: This study uses analytic morphomics to define the Morphomic Malnutrition Score (MMS), a robust screening tool for severe malnutrition. Methods: The study population $(n=643)$ consisted of 2 cohorts: 1) 124 emergency department patients diagnosed with severe malnutrition by a registered dietitian (RD) and an available computed tomography (CT) scan within 2 days of RD evaluation, and 2) 519 adult kidney donor candidates to represent a healthy cohort. Body composition markers of muscle area and abdominal adiposity were measured from patient $\mathrm{CT}$ scans using analytic morphomic assessment, and then converted to sex- and age-adjusted percentiles using the Reference Analytic Morphomics Population (RAMP). RAMP consists of 6000 patients chosen to be representative of the general population. The combined cohort was then randomly divided into training $(\mathrm{n}=453)$ and validation $(\mathrm{n}=190)$ sets. MMS was derived using logistic regression. The model coefficients were transformed into a score, normalized from 0 to $10(10=$ most severe). Results: Severely malnourished patients had lower amounts of muscle and fat than kidney donors, specifically for dorsal muscle group area at the twelfth thoracic vertebral level $(P<0.001)$, psoas muscle area at the fourth lumbar vertebral level $(P<0.001)$, and subcutaneous fat area at the third lumbar vertebral level $(P<0.001)$ - all parameters in MMS. MMS for severely malnourished patients was higher than kidney donors $(7.7 \pm 2.2$ vs $3.8 \pm 2.0$, respectively; $P$-value $<0.001)$. An MMS $>6.1$ was accurate in determining nutrition diagnosis ( $82.1 \%$ sensitivity; $88.3 \%$ specificity; $85.2 \%$ balanced accuracy). Conclusions: MMS provides an evidence-based, granular assessment to distinguish severely malnourished adults from a healthy population. (JPEN J Parenter Enteral Nutr. 2018;42:12631271)
\end{abstract}

\section{Keywords}

body composition; malnutrition; morphomics; screening tool

\section{Clinical Relevancy Statement}

A malnutrition score using patient-specific measures of body composition measured from computed tomography scans provides a standardized screening criterion for severe malnutrition in adults. This novel screening tool utilizes robust surrogates for fat and muscle loss to produce a highly sensitive and specific test for detecting severe malnutrition. This work is clinically relevant for clinicians who recognize malnutrition as a risk factor for poor clinical outcomes and who are aiming for a more efficient and reproducible method to screen for patients at true risk.

From the ${ }^{1}$ Morphomic Analysis Group, University of Michigan, Ann Arbor, Michigan, USA; ${ }^{2}$ Department of Patient Food and Nutrition Services, Ann Arbor, Michigan, USA; ${ }^{3}$ Department of Emergency Medicine, University of Wisconsin, Madison, Wisconsin, USA; ${ }^{4}$ University of Michigan, Ann Arbor, Michigan, USA $;{ }^{5}$ Department of Surgery, Michigan Medicine, Ann Arbor, Michigan, USA; ${ }^{6}$ Department of Medicine, Michigan Medicine, Ann Arbor, Michigan, USA; and the ${ }^{7}$ Gastroenterology Section, VA Ann Arbor Healthcare System, Ann Arbor, Michigan, USA.

Financial disclosure: None declared.

Conflicts of interest: Stewart C. Wang is listed as an inventor on U.S. Patent \#US 20140064583. All other authors have none to declare.

Received for publication December 11, 2017; accepted for publication March 21, 2018.

This article originally appeared online on May 22, 2018.

Corresponding Author:

Stewart C. Wang, MD, PhD, FACS, Morphomic Analysis Group, 1150 W. Medical Center Drive, 3328 Medical Science 1-SPC 5677, Ann Arbor, Michigan 48109-5677.

Email: stewartw@med.umich.edu 


\section{Introduction}

Malnutrition is "an acute, subacute or chronic state of nutrition, in which a combination of varying degrees of overnutrition or undernutrition with or without inflammatory activity have led to a change in body composition and diminished function."1(p6) Malnutrition contributes to increased morbidity and mortality in hospitalized adult patients, including increased risk for developing nosocomial infection, pressure injury, and other complications. ${ }^{2}$ Additionally, malnutrition is associated with longer hospital length of stay, increased hospital readmission, and increased healthcare costs. ${ }^{2}$

The incidence and prevalence of malnutrition differ between patient populations and healthcare institutions and vary based on the assessment tools used to identify malnutrition across care settings. ${ }^{3-5}$ The incidence and prevalence of malnutrition are somewhat difficult to determine due to a lack of objective tests that can be used to definitively define and substantiate the malnutrition diagnosis. ${ }^{3-5}$ Traditional malnutrition indices often fail to consider the extent to which inflammation and illness contribute to the development and progression of malnutrition..$^{3-5}$

In 2012, the American Society for Parenteral and Enteral Nutrition (ASPEN) and the Academy of Nutrition and Dietetics (Academy) jointly published consensus recommendations for the identification and documentation of adult malnutrition (undernutrition). ${ }^{4}$ The consensus statement established standardized criteria for severe and nonsevere (moderate) malnutrition within 6 domains: energy intake, weight loss, loss of muscle mass, loss of subcutaneous fat, localized or generalized fluid that may mask weight loss, and diminished functional status measured by handgrip strength. The recommendations outlined etiology-based definitions for malnutrition based on acute illness/injury, chronic disease, and starvation. ${ }^{6}$

The consensus statement aimed to alleviate compliance challenges in diagnosing and documenting adult malnutrition, using a standardized set of characteristics to generate consistent and reproducible results. These recommendations differ from traditional and historic definitions of malnutrition in identifying malnutrition based on etiology, considering multiple manifestations of undernutrition, and discriminating between nonsevere and severe malnutrition. ${ }^{4,5}$

Differentiating between mild, moderate, and severe changes in body composition, as outlined in this paper, can be challenging and subject to interobserver bias. The accuracy and reliability of using muscle loss, fat loss, fluid accumulation, and handgrip strength to diagnose malnutrition may be confounded by other variables specific to a patient's case, condition, or overall treatment plan. Definitions of mild malnutrition were not included in this paper. Discrepancy remains between evidence-based best practice recommendations for malnutrition identification. ${ }^{7}$ Overall, rigorous validation of the ASPEN/Academy consensus recommendations have not been published, and opportunities exist to develop methods that can objectively measure and quantify changes in body composition over time.

Analytic morphomics utilizes computed tomography (CT) scans to provide patient-specific body composition markers and may provide the standardization that is lacking in conventional diagnostics. Previous investigators have associated morphomic measurements of muscle and fat with mortality and complications across varying clinical populations. ${ }^{8-13}$ We used analytic morphomics measures of muscle and fat to develop a Morphomic Malnutrition Score (MMS). We hypothesize that the novel score can serve as a robust screening tool for severe malnutrition in adults.

\section{Methods}

\section{Study Population}

MMS is a regression coefficient-based score that was trained and validated on a retrospectively collected study population that consisted of both severely malnourished and normal patient populations (Figure 1).

The severely malnourished cohort $(n=124)$ included the following: adult patients admitted from the Michigan Medicine emergency department (ED) between 2014 and 2016 who 1) were administered a nutrition assessment by a registered dietitian (RD) during their encounter; 2) had an RD note with a nutrition diagnosis of severe malnutrition; and 3) had a CT scan within 2 days of RD evaluation that included complete imaging from the tenth thoracic (T10) through fourth lumbar (L4) vertebral level. Prior to being included in the analysis, each patient's medical record was reviewed retrospectively by a Michigan Medicine RD to confirm the clinical diagnosis of severe malnutrition satisfied current Michigan Medicine criteria. Malnutrition at Michigan Medicine was identified using a standard set of criteria. The criteria include characteristics for severe and nonsevere (moderate) malnutrition in specific domains: weight loss over time, body mass index (BMI), dietary inadequacies, and physical signs and symptoms (muscle loss and fat loss). Patients were classified as severely malnourished based on the identification of 2 or more characteristics from 2 different domains (Table 1). Patient age, sex, BMI, and serum albumin level were retrospectively collected from electronic medical records. BMI at the time of nutrition assessment was recorded by the RD; serum albumin level was obtained proximal to RD evaluation date.

The normal cohort of adult kidney donor candidates ( $n=519)$ with an appropriate CT scan between 2000 and 2010 was selected to represent a clinically healthy population. Despite these patients not having undergone an RD evaluation, the selection criteria for donor candidates ${ }^{14,15}$ 


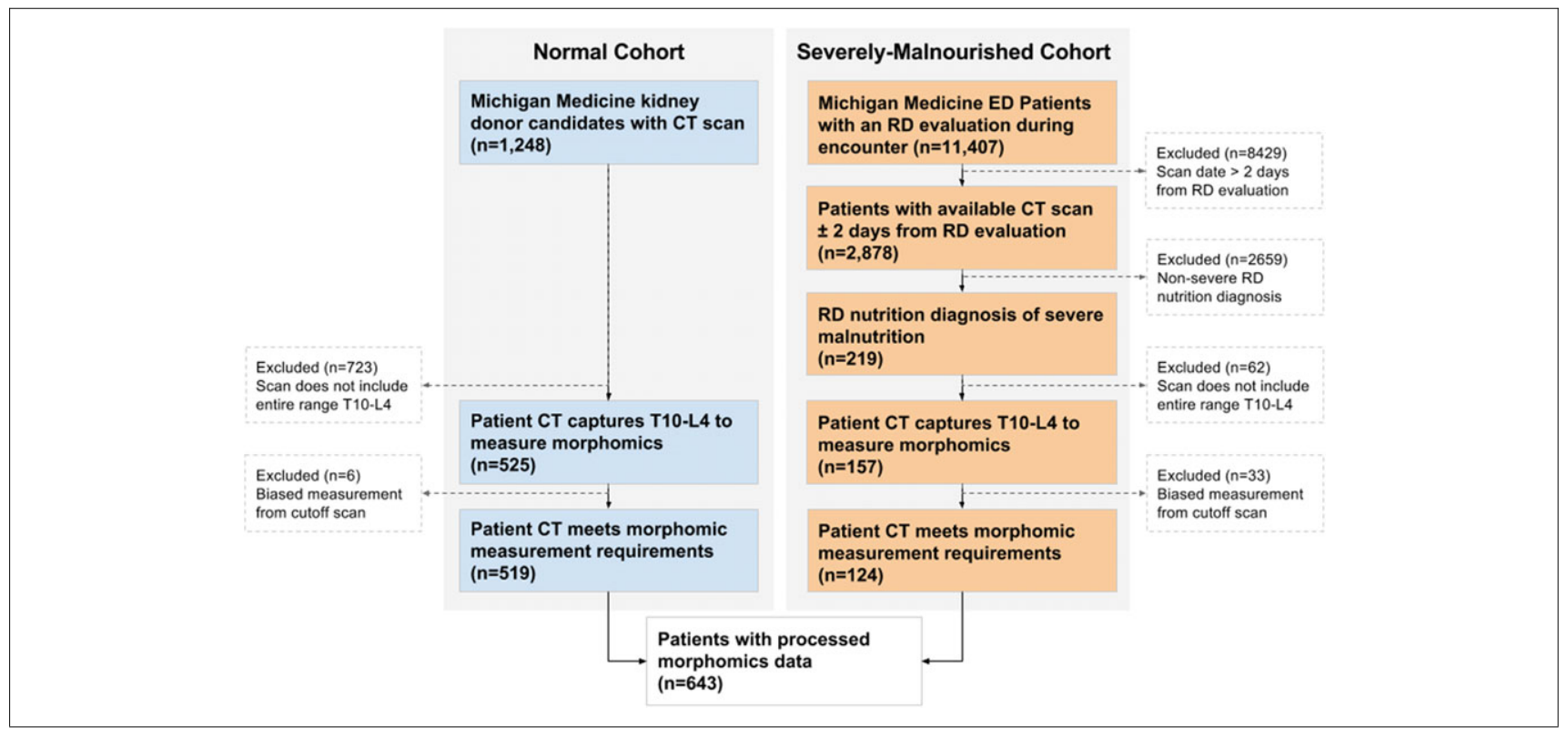

Figure 1. Study population selection flow chart. CT, computed tomography; ED, emergency department; L, lumbar, RD, registered dietitian; $\mathrm{T}$, thoracic.

substantiated a nutrition diagnosis of "normal." Patient age, sex, BMI, and serum albumin level were retrospectively collected from electronic medical records closest to patient scan date; inclusion criteria were the same as well. Both cohorts were combined to form our study population $(n=643)$.

\section{Analytic Morphomics}

In this study, cross-sectional areas of dorsal muscle group (DMA), total psoas muscle (TPA), and subcutaneous fat (SFA) were measured between the T10 through L4 vertebral levels to observe trends along the torso. The in-depth methodology on how these morphomic parameters were measured has been covered extensively in our previous works. $^{8-13}$ The CT image processing was performed with semi-automated algorithms in MatLab 13.0 (MathWorks, Natick, MA). ${ }^{16}$ Initially, scans were labeled at each vertebral level to provide anatomical landmarks for subsequent measurements. Cross-sectional area of L3 SFA, T12 DMA, and L4 TPA were selected from available measurements (Figure 2). The selection of these measures was based on previous works demonstrating the clinical relevance of these CT measures of fat and muscle in clinical populations. The area measures were then converted into sexand age-matched percentiles based on Reference Analytic Morphomic Population (RAMP) growth curves. ${ }^{17}$

\section{$R A M P$}

RAMP consists of approximately 6000 patients chosen to be representative of the general population and includes both healthy and unhealthy individuals. Chest, abdomen, and pelvis CT scans were collected from patients aged 191 years, at Michigan Medicine, who were scanned primarily for trauma indications. Quantile regression was performed on each morphomic factor vs age separately for males and females to generate growth curves corresponding to the 5th, 25th, 50th, 75th, and 95th percentiles (Figure 3). This detailed approach to body composition enables the conversion of individual CT body composition measures into age- and sex-matched percentiles. Any individual with a value above the 95 th or below the 5 th percentile was assigned to the 95 th or 5 th, respectively.

\section{Statistical Methods}

The study population was randomly split into a training set $(\mathrm{n}=453,70 \%)$ and validation set $(\mathrm{n}=190,30 \%)$. Univariate tests were performed on the training set to assess statistical significance between patient factors and nutrition diagnosis. $T$-tests were used to compare means of continuous variables; Fisher exact test was used to compare proportions of binary variables between severely malnourished and normal patients. Candidate parameters of the morphomics score were those shown to be associated with patient outcomes in previous studies ${ }^{8-13}$ and demonstrated both a significant relationship and good discrimination ability with nutrition diagnosis, as measured by $P$-value and area under the receiver operating characteristic curve (AUROC), respectively. Using the training set, a multivariable logistic regression model with nutrition diagnosis as the dependent variable was generated with the selected parameters using elastic 
Table 1. Michigan Medicine Adult Malnutrition Diagnosis Guidelines.

\begin{tabular}{|c|c|c|c|}
\hline & ICD-10 Code & $\begin{array}{l}\text { Malnutrition } \\
\text { (Moderate) } \\
\text { E44.0 }\end{array}$ & $\begin{array}{c}\text { Malnutrition (Severe, } \\
\text { Protein-Calorie) } \\
\text { E43 }\end{array}$ \\
\hline \multirow[t]{5}{*}{ Domain } & Weight loss & $\begin{array}{l}\text { Significant weight loss: } \\
1 \%-2 \% \text { in } 1 \text { week } \\
5 \% \text { in } 1 \text { month } \\
7.5 \% \text { in } 3 \text { months } \\
10 \% \text { in } 6 \text { months } \\
20 \% \text { in } 12 \text { months }\end{array}$ & $\begin{array}{l}\text { Severe weight loss: } \\
>2 \% \text { in } 1 \text { week } \\
>5 \% \text { in } 1 \text { month } \\
>7.5 \% \text { in } 3 \text { months } \\
>10 \% \text { in } 6 \text { months } \\
>20 \% \text { in } 12 \text { months }\end{array}$ \\
\hline & BMI & N/A & $\mathrm{BMI}<18.5$ \\
\hline & $\begin{array}{l}\text { Dietary } \\
\text { inadequacies }\end{array}$ & $\begin{array}{l}>7 \text { days with a nutrient intake of } \\
\leq 75 \% \text { of total estimated energy } \\
\text { requirements } \\
\text { or } \\
>7 \text { days with a nutrient intake of } \\
\leq 75 \% \text { of baseline/usual intake }\end{array}$ & $\begin{array}{l}\geq 1 \text { month with intake of } \leq 50 \% \\
\text { of total estimated energy } \\
\text { requirement } \\
\text { or } \\
\geq 1 \text { month with intake of } \leq 50 \% \\
\quad \text { of baseline/usual intake }\end{array}$ \\
\hline & Physical findings & $\begin{array}{l}\text { Mild loss of muscle mass } \\
\text { Mild loss of subcutaneous fat }\end{array}$ & $\begin{array}{l}\text { Moderate or severe loss of muscle } \\
\text { mass } \\
\text { Moderate or severe loss of } \\
\text { subcutaneous fat } \\
\text { Delayed wound healing }\end{array}$ \\
\hline & Functional status & N/A & $\begin{array}{l}\text { Markedly reduced hand grip } \\
\text { strength ( }>2 \text { SD below mean) }\end{array}$ \\
\hline
\end{tabular}

A minimum of 2 characteristics from 2 different domains is required for the diagnosis of malnutrition.

Clinical judgement should be used when using this table for the identification of malnutrition.

BMI, body mass index; ICD-10, 10th Revision of the International Classification of Diseases; N/A, not applicable.

net regularization to mitigate the risks of overfitting and collinearity. ${ }^{18}$ To create a more intuitive evaluation metric, the final regression equation was transformed into a score (MMS) normalized to a range of $0.0-10.0$, rounded to the nearest 10 th, with 10.0 being the most severe degree of malnutrition.

The optimal MMS cutoff to distinguish severe malnutrition from a normal diagnosis was selected to maximize balanced accuracy [(sensitivity + specificity)/2] The ability of this method to correctly classify patients as severely malnourished or normal was assessed by comparing its sensitivity, specificity, and balanced accuracy in the validation set to those achieved by using the clinical criteria of low BMI $\left(<19 \mathrm{~kg} / \mathrm{m}^{2}\right)$ and low serum albumin level $(<3.5 \mathrm{~g} / \mathrm{dL})$, using the RD diagnosis as the benchmark classification.

Statistical significance was determined by an $\alpha$-level of 0.05 , and statistical analysis was performed in $\mathrm{R}$ version 3.4.2. ${ }^{19}$

This Health Insurance Portability and Accountability Act-compliant study was approved by the Medical School Institutional Review Board at the University of Michigan. The requirement for informed consent was waived.

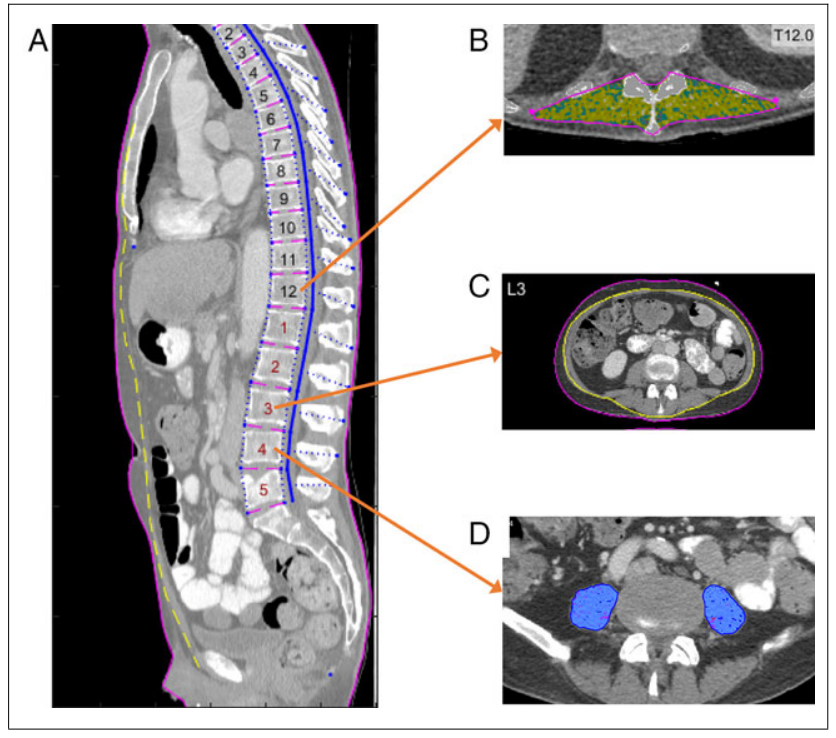

Figure 2. Example of CT scan used in analytic morphomics assessment. (A) Individual vertebrae were labeled.

(B) Cross-sectional area of dorsal muscle group at T12,

(C) subcutaneous fat (region between purple and yellow lines) at L3, and (D) psoas muscles at L4 were measured. CT, computed tomography; L, lumbar; T, thoracic. 


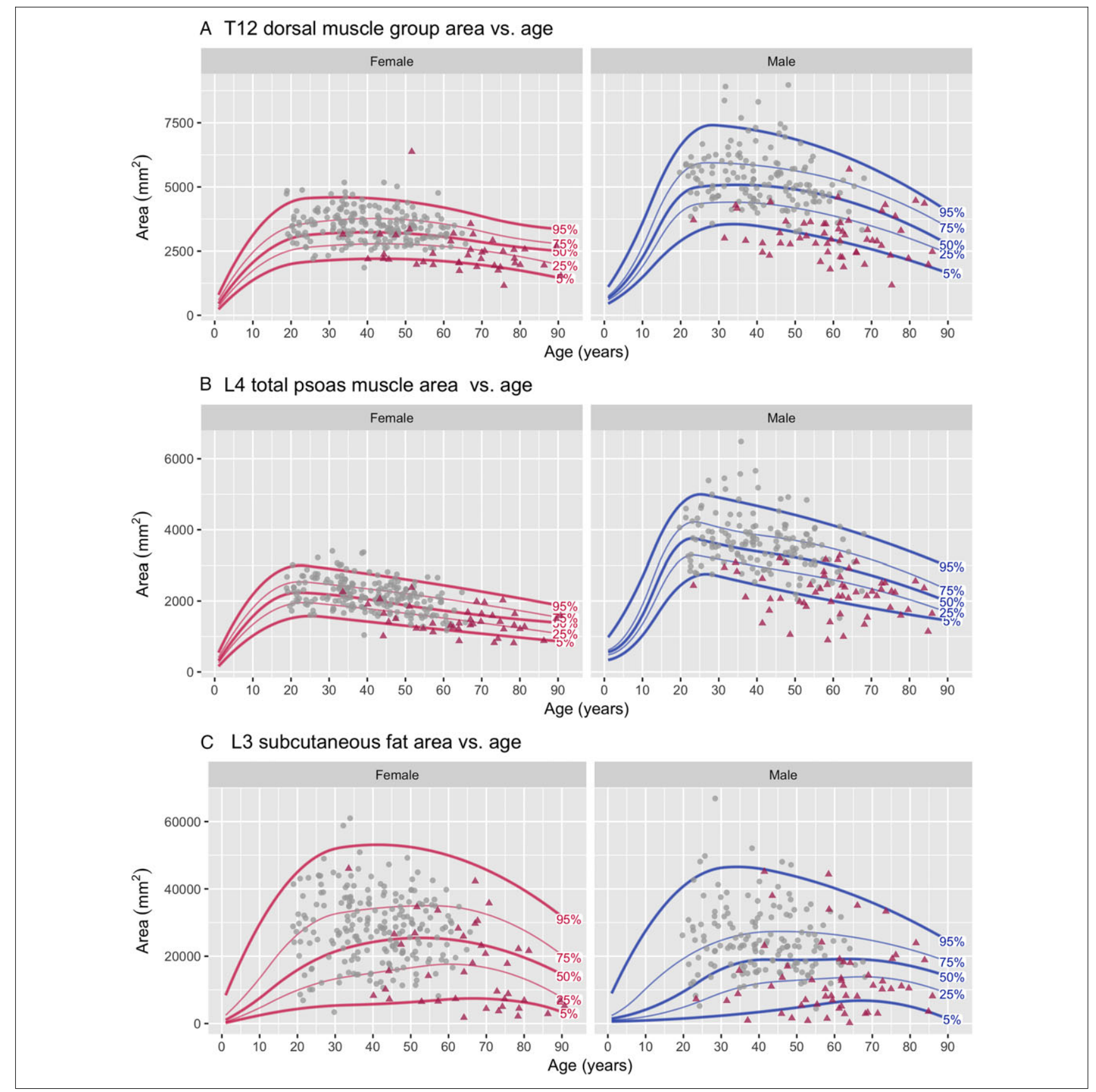

Figure 3. Reference analytic morphomics population growth curves with 5 th, 25 th, 50 th, 75 th, and 95 th percentiles by age and (A) sex for dorsal muscle group; (B) total psoas muscle; and (C) subcutaneous fat. The training set severely malnourished (maroon triangles) and normal (gray circles) cohorts are overlaid atop the growth curves, showing their relative distributions. L, lumbar; T, thoracic.

\section{Results}

\section{Study Population}

$T$-tests and Fisher exact test showed significant differences $(P<0.001)$ between normal and severely malnourished patients in all observed variables (Table 2). Furthermore, univariate area under the curve (AUC) values showed better discriminating ability using body composition percentiles when compared against the clinical predictors of low BMI and low serum albumin level. Compared with the normal cohort, the severely malnourished cohort was older; and a greater proportion were male, had low BMI, and had 
Table 2. Summary Statistics Comparing Severely Malnourished and Normal Cohorts.

\begin{tabular}{|c|c|c|c|c|c|c|c|c|c|}
\hline \multirow[b]{2}{*}{ Variable } & \multirow[b]{2}{*}{ Vertebra } & \multicolumn{3}{|c|}{ Severely Malnourished } & \multicolumn{3}{|c|}{ Normal } & \multirow[b]{2}{*}{$P$-Value } & \multirow[b]{2}{*}{ AUC } \\
\hline & & $\mathrm{N}$ & Mean & $\mathrm{SD}$ & $\mathrm{N}$ & Mean & $\mathrm{SD}$ & & \\
\hline \multicolumn{10}{|l|}{ Training Set } \\
\hline Age (years) & & 96 & 62.0 & 14.3 & 357 & 40.8 & 11.6 & $<.001$ & 0.87 \\
\hline Female & & 96 & $41.7 \%$ & & 357 & $58.3 \%$ & & $<.004$ & 0.58 \\
\hline $\begin{array}{l}\text { Serum albumin level low } \\
(<3.5 \mathrm{~g} / \mathrm{dL})\end{array}$ & & 93 & $52.7 \%$ & & 354 & $0.6 \%$ & & $<.001$ & 0.76 \\
\hline BMI low $\left(<19 \mathrm{~kg} / \mathrm{m}^{2}\right)$ & & 86 & $24.4 \%$ & & 351 & $0.3 \%$ & & $<.001$ & 0.38 \\
\hline Malnutrition score & & 96 & 7.7 & 2.2 & 357 & 3.8 & 2.0 & $<.001$ & 0.89 \\
\hline DMA $_{\text {centile }}$ & $\mathrm{T} 10$ & 96 & 27.5 & 23.6 & 357 & 59.1 & 26.6 & $<.001$ & 0.81 \\
\hline DMA $_{\text {centile }}$ & $\mathrm{T} 11$ & 96 & 23.3 & 22.4 & 357 & 61.6 & 25.2 & $<.001$ & 0.87 \\
\hline$D M A_{\text {centile }}$ & $T 12$ & 96 & 21.9 & 23.7 & 357 & 59.6 & 24.8 & $<.001$ & 0.87 \\
\hline DMA $_{\text {centile }}$ & L1 & 96 & 22.7 & 22.6 & 357 & 54.4 & 25.9 & $<.001$ & 0.83 \\
\hline $\boldsymbol{T P} \boldsymbol{A}_{\text {centile }}$ & $L 4$ & 96 & 27.3 & 24.1 & 357 & 58.3 & 26.6 & $<.001$ & 0.81 \\
\hline SFA $_{\text {centile }}$ & $\mathrm{T} 10$ & 96 & 32.0 & 29.1 & 357 & 59.9 & 23.6 & $<.001$ & 0.77 \\
\hline SFA $_{\text {centile }}$ & $\mathrm{T} 11$ & 96 & 31.1 & 29.6 & 357 & 61.5 & 23.1 & $<.001$ & 0.79 \\
\hline SFA $_{\text {centile }}$ & $\mathrm{T} 12$ & 96 & 31.3 & 30.2 & 357 & 62.6 & 23.0 & $<.001$ & 0.79 \\
\hline SFA $_{\text {centile }}$ & $\mathrm{L} 1$ & 96 & 31.7 & 30.6 & 357 & 63.6 & 22.9 & $<.001$ & 0.79 \\
\hline SFA $_{\text {centile }}$ & $\mathrm{L} 2$ & 96 & 31.8 & 30.0 & 357 & 62.7 & 22.7 & $<.001$ & 0.79 \\
\hline $\boldsymbol{S F} \boldsymbol{A}_{\text {centile }}$ & $L 3$ & 96 & 30.7 & 28.8 & 357 & 62.0 & 22.7 & $<.001$ & 0.80 \\
\hline $\mathrm{SFA}_{\text {centile }}$ & $\mathrm{L} 4$ & 96 & 30.1 & 28.5 & 357 & 60.3 & 23.0 & $<.001$ & 0.79 \\
\hline \multicolumn{10}{|l|}{ Validation Set } \\
\hline Age & & 28 & 57.1 & 16.9 & 162 & 40.6 & 11.2 & $<.001$ & 0.78 \\
\hline Female & & 28 & $54 \%$ & & 162 & $66 \%$ & & 0.208 & 0.56 \\
\hline $\begin{array}{l}\text { Serum albumin level low } \\
(<3.5 \mathrm{~g} / \mathrm{dL})\end{array}$ & & 27 & $63 \%$ & & 157 & $0 \%$ & & $<.001$ & 0.81 \\
\hline BMI low $\left(<19 \mathrm{~kg} / \mathrm{m}^{2}\right)$ & & 24 & $42 \%$ & & 159 & $0 \%$ & & $<.001$ & 0.29 \\
\hline Malnutrition score & & 28 & 8.1 & 1.9 & 162 & 3.5 & 2.0 & $<.001$ & 0.94 \\
\hline DMA $_{\text {centile }}$ & $\mathrm{T} 10$ & 28 & 26.8 & 28.2 & 162 & 63.5 & 23.6 & $<.001$ & 0.84 \\
\hline DMA $_{\text {centile }}$ & $\mathrm{T} 11$ & 28 & 22.8 & 26.7 & 162 & 66.0 & 24.3 & $<.001$ & 0.88 \\
\hline$D M A_{\text {centile }}$ & $T 12$ & 28 & 22.8 & 27.5 & 162 & 63.7 & 24.7 & $<.001$ & 0.87 \\
\hline DMA $_{\text {centile }}$ & L1 & 28 & 25.5 & 29.9 & 162 & 58.1 & 25.1 & $<.001$ & 0.81 \\
\hline $\boldsymbol{T P} \boldsymbol{A}_{\text {centile }}$ & $L 4$ & 28 & 22.5 & 21.8 & 162 & 63.5 & 24.6 & $<.001$ & 0.89 \\
\hline SFA $_{\text {centile }}$ & $\mathrm{T} 10$ & 28 & 21.0 & 15.3 & 162 & 61.4 & 23.3 & $<.001$ & 0.91 \\
\hline SFA $_{\text {centile }}$ & T11 & 28 & 20.6 & 15.7 & 162 & 62.1 & 22.4 & $<.001$ & 0.92 \\
\hline $\mathrm{SFA}_{\text {centile }}$ & $\mathrm{T} 12$ & 28 & 21.3 & 15.9 & 162 & 63.5 & 21.8 & $<.001$ & 0.93 \\
\hline SFA $_{\text {centile }}$ & $\mathrm{L} 1$ & 28 & 21.1 & 16.2 & 162 & 64.1 & 21.0 & $<.001$ & 0.94 \\
\hline SFA $_{\text {centile }}$ & $\mathrm{L} 2$ & 28 & 20.2 & 15.3 & 162 & 64.3 & 20.6 & $<.001$ & 0.95 \\
\hline $\mathbf{S F A} \mathbf{A}_{\text {centile }}$ & $\mathbf{L 3}$ & 28 & 18.6 & 14.3 & 162 & 63.4 & 21.0 & $<.001$ & 0.95 \\
\hline SFA $_{\text {centile }}$ & L4 & 28 & 18.1 & 14.3 & 162 & 61.4 & 22.2 & $<.001$ & 0.94 \\
\hline
\end{tabular}

Variables used in the malnutrition score in bold.

AUC, area under the curve; BMI, body mass index; DMA, dorsal muscle group; L, lumbar; SFA, subcutaneous fat; T, thoracic; TPA, total psoas muscle.

low serum albumin level. The severely malnourished cohort had lower percentiles across all measured vertebra levels for DMA, TPA, and SFA.

\section{Malnutrition Scoring and Classification}

Multivariable logistic regression demonstrated that low muscle and fat area percentiles were associated with severe malnutrition (regression coefficients, L3 SFA centile: -0.025 ; L4 TPA centile $:-0.020 ; \mathrm{T} 12 \mathrm{DMA}_{\text {centile }}$ : -0.036). After normalization, the final MMS equation was:

$$
\begin{aligned}
M M S= & 10.55-\left(.0343 * S F A_{\text {centile }}\right)-\left(.0274 * T P A_{\text {centile }}\right) \\
& -\left(.0494 * D M A_{\text {centile }}\right)
\end{aligned}
$$

Patients with severe malnutrition had higher MMS than normal patients $(7.7 \pm 2.2$ vs $3.8 \pm 2.0$, respectively; $P$ value $<0.001$ ). MMS had an AUROC of 0.89 in the training set and 0.94 in the validation set (Figure 4a). A cutoff score of MMS > 6.1 maximized balanced accuracy 


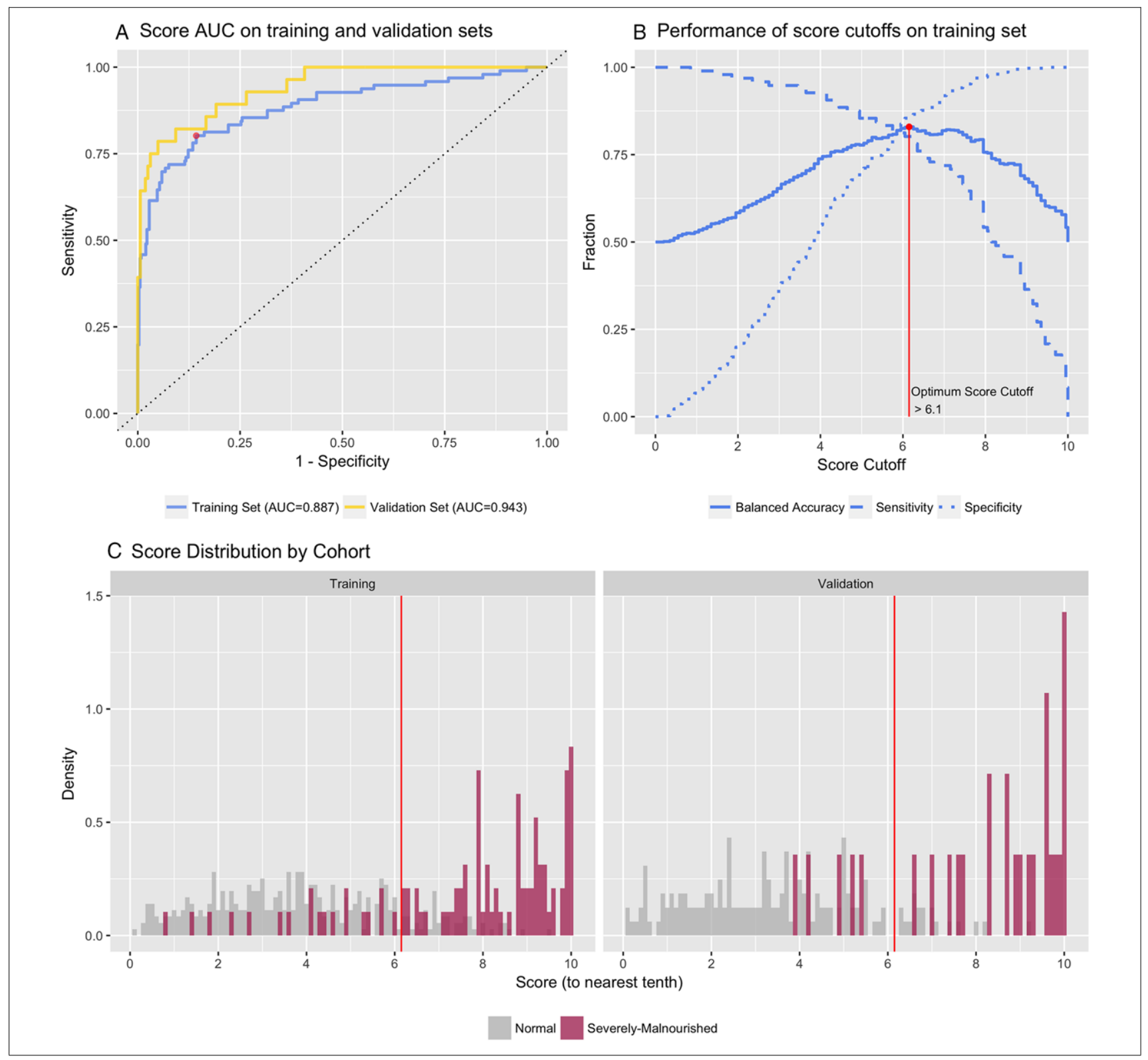

Figure 4. (A) Sensitivity, specificity, and balanced accuracy of various score cutoffs on the training set, showing the optimum score cutoff at 6.1. (B) Performance of AUROC curve of multivariable logistic regression model performance on training and validation sets. (C) Distribution of scores for normal vs severely malnourished cohorts in both training and validation sets. AUC, area under the curve.

in the training set and was selected to distinguish between a nutrition diagnosis of severe and normal (Figure $4 b$ ). The distribution of scores in the training set and validation set were similar; however, the improved AUC was attributed to the paucity of low scores in the severely malnourished cohort in the validation set (Figure 4c). In the validation set, low BMI had a sensitivity of $41.7 \%$, specificity of $100 \%$, and balanced accuracy of $70.8 \%$; low serum albumin level had a sensitivity of $63 \%$, specificity of $100 \%$, and balanced accuracy of $81.5 \%$; and MMS $>6.1$ produced sensitivity of
$82.1 \%$, specificity of $88.3 \%$, and balanced accuracy of $85.2 \%$ (Table 3).

\section{Discussion}

In this study, we present a novel standardized screening tool for severe malnutrition that utilizes sex- and age-adjusted reference percentiles of muscle area and abdominal adiposity incidentally imaged by $\mathrm{CT}$ performed for unrelated clinical indications. Lower percentiles of TPA area, DMA 
Table 3. Performance of High Malnutrition Score, Low Serum Albumin Level, and Low BMI in Classifying Patients as Severely Malnourished vs Registered Dietitian Diagnosis.

\begin{tabular}{llccc}
\hline Dataset & Model & Specificity & Sensitivity & Balanced Accuracy \\
\hline Training & High MMS $(>6.1)$ & $85.7 \%$ & $80.2 \%$ & $83.0 \%$ \\
& Low serum albumin level $(<3.5 \mathrm{~g} / \mathrm{dL})$ & $99.4 \%$ & $52.7 \%$ & $76.1 \%$ \\
& Low BMI $\left(<19 \mathrm{~kg} / \mathrm{m}^{2}\right)$ & $99.7 \%$ & $24.4 \%$ & $62.1 \%$ \\
Validation & High MMS $(>6.1)$ & $88.3 \%$ & $82.1 \%$ & $85.2 \%$ \\
& Low serum albumin level $(<3.5 \mathrm{~g} / \mathrm{dL})$ & $100.0 \%$ & $63.0 \%$ & $81.5 \%$ \\
& Low BMI $\left(<19 \mathrm{~kg} / \mathrm{m}^{2}\right)$ & $100.0 \%$ & $41.7 \%$ & $70.8 \%$ \\
\hline
\end{tabular}

BMI, body mass index; MMS, Morphomic Malnutrition Score.

area, and SFA demonstrated a strong association with a severe malnutrition diagnosis. The multivariate model from which MMS was derived was internally validated and demonstrated improved accuracy in classifying severely malnourished vs normal patients when compared with low BMI or low serum albumin level alone. Scores for severely malnourished patients were significantly higher than patients diagnosed as normal. Furthermore, the cutoff value for severe malnutrition generated a highly sensitive diagnostic test. Internal validation of the score cutoff generated the highest sensitivity when compared against indicators for low BMI and low serum albumin level. Within this context, high sensitivity is most useful for MMS to avoid incorrectly refuting the presence of a potentially treatable risk factor such as malnutrition.

The recent consensus statement from ASPEN and the Academy proposed a set of characteristics to improve malnutrition identification. Their criteria included intake, weight loss, loss of muscle mass, loss of subcutaneous fat, localized or generalized fluid, and diminished functional status to provide an etiology-based approach to make malnutrition diagnostics more reproducible and easier to define. BMI was among the parameters in the Michigan RD nutrition assessment. BMI is recognized as a widely used indicator for obesity and nutrition status; however, previous studies have shown that it is unable to discern lean mass content from body fat mass. ${ }^{20}$ Albumin is another common diagnostic criteria that previous investigators-including ASPEN and the Academy-have demonstrated to be an insensitive and nonspecific indicator of nutrition status. ${ }^{4}$ Neither BMI nor serum albumin level alone are reasonable screening criteria for severe malnutrition; however, they were the available criteria that were retrospectively available for all study patients. Because low BMI is included in the existing RD diagnosis, we would expect it to perform reasonably well. However, serum albumin level is not included in the RD diagnosis and was only included due to its previous use by other investigators. ${ }^{21,22}$ Like those investigators, we do not recommend using low serum albumin level as an indicator of malnutrition in hospitalized patients because serum albumin level is an acute phase reactant and plasma levels are decreased in a number of disease states unrelated to nutrition status such as injury, inflammation, sepsis, fluid shifts and anasarca, and synthetic liver dysfunction. MMS aims to inform the development of an objective screening protocol by providing a quantitative assessment of muscle and fat, independent of factors such as BMI and serum albumin level, to better characterize body changes caused by malnutrition.

\section{Conclusions}

There are limitations to this study. First, this is a retrospective study and is subject to biases of such methodology. Our study population was also from a single institution. Although we cannot argue that it is representative of a general ED patient population, the opportunity to produce a novel diagnostic criterion for severe malnutrition using reproducible methods was deemed worthwhile to work within the shortcomings of our data. Our nutrition diagnosis of kidney donor candidates as "normal" has not been validated by clinicians or health centers outside of this work. Evaluation for kidney donation at Michigan Medicine is available to those who are genuinely willing to donate; physically fit; in good general health; and free from diabetes, cancer, kidney disease, and heart disease. The predonation evaluation includes assessment by a nephrologist, transplant surgeon, and a social worker, and candidates only receive a CT if they have completed their evaluation and were deemed an excellent candidate to donate a kidney. Classifying these successful candidates as nutritionally "normal" was a clinically based decision that involved both the RD and the physicians. The exclusion of all other ED patients with a nonsevere (eg, moderate, none) nutrition diagnosis presents bias. However, the characteristics of severe malnutrition had the most objective criteria to substantiate the diagnosis. Furthermore, it was our objective to first assess whether our novel markers of muscle and fat could quantitatively distinguish a healthy patient from the severely malnourished population.

Future works will focus on investigating clinical populations where malnutrition is prevalent and CT imaging is 
part of the treatment protocol. Calculating MMS of other populations and associating it with clinical outcomes can inform the link between malnutrition and poor outcomes. Patient claims data is also desirable in order to determine whether an MMS screen positive for severe malnutrition is associated with higher episode costs. MMS serves as a robust indicator of a widely recognized risk factor that is not clearly defined, yet likely modifiable if administered the appropriate regimen. Furthermore, MMS utilizes patientspecific data measured from CT scans performed for other medical indications, making implementation simple and scalable for screening to identify patients who would benefit from RD assessment and intervention for malnutrition.

\section{Acknowledgment}

The authors would like to thank Caryn Boyd for her assistance in procuring ED raw data elements.

\section{Statement of Authorship}

C. Lee, B. A. Derstine, J. M. Glazer, J. Cherry-Bukowiec, G. L. Su, and S. C. Wang equally contributed to the conception and design of the research; J. M. Glazer, R. Goulson, and A. Rajasekaran contributed to the acquisition of data; J. Cherry-Bukowiec and E. Raymond contributed to acquisition and interpretation of the data; G. L. Su, contributed to interpretation of the data; C. Lee, B. A. Derstine, and S. C. Wang contributed to acquisition, analysis, and interpretation of the data. C. Lee, E. Raymond, and B. A. Derstine drafted the manuscript. All authors critically revised the manuscript, agree to be fully accountable for ensuring the integrity and accuracy of the work, and read and approved the final manuscript.

\section{References}

1. A.S.P.E.N. Board of Directors and Clinical Practice Committee. Definition of Terms, Style, and Conventions Used in A.S.P.E.N. Board of Directors- Approved Documents. Available at: http://www.nutrit ioncare.org/uploadedFiles/Home/Guidelines_and_Clinical_Practice/ DefinitionsStyleConventions.pdf. Accessed November 20, 2017.

2. National Alliance for Infusion Therapy and the American Society for Parenteral and Enteral Nutrition Public Policy Committee and Board of Directors. Disease-related malnutrition and enteral nutrition therapy: a significant problem with a cost-effective solution. Nutr Clin Pract 2010;25:548-554.

3. White JV, Stotts N, Jones SW, Granieri E. Managing postacute malnutrition (undernutrition) risk. JPEN J Parenter Enteral Nutr 2013;37:816-823.
4. White JV, Guenter P, Jensen G, Malone A, Schofield M; the Academy Malnutrition Work Group, the A.S.P.E.N Board of Directors. Consensus statement: Academy of Nutrition and Dietetics and American Society for Parenteral and Enteral Nutrition: characteristics recommended for the identification and documentation of adult malnutrition (undernutrition). JPEN J Parenter Enteral Nutr 2012;36:275-283.

5. Tappenden KA, Quatrara B, Parkhurst, ML, et al. Critical role of nutrition in improving quality of care: an interdisciplinary call to action to address hospital malnutrition. JPEN J Parenter Enter Nutr 2013;37:482-489.

6. Jensen GL, Bistrian B, Roubenoff R, Heimburger DC. Malnutrition syndromes: a conundrum vs continuum. JPEN J Parenter Enteral Nutr 2009:33:710.

7. Cederholm T, Bosaeus I, Barazzoni R, et al. Diagnostic criteria for malnutrition: an ESPEN consensus statement. Clin Nutr ESPEN 2015;34:335-340.

8. Englesbe MJ, Patel SP, He K, et al. Sarcopenia and mortality after liver transplantation. J Am Coll Surg 2010;211:271-278.

9. Englesbe MJ, Lee JS, He K, et al. Analytic morphomics, core muscle size, and surgical outcomes. Ann Surg 2012;256:255-261.

10. Lee CS, Cron DC, Terjimanian MN, et al. Dorsal muscle group area and surgical outcomes in liver transplantation. Clin Transplant 2014;28:1092-1098

11. Canvasser LD, Mazurek AA, Cron DC, et al. Paraspinous muscle as a predictor of surgical outcome. J Surg Res 2014;192:76-81.

12. Lee JS, Terjimanian MN, Tishberg LM, et al. Surgical site infection and analytic morphometric assessment of body composition in patients undergoing midline laparotomy. J Am Coll Surg 2011;213:236-244.

13. Stidham RW, Waljee AK, Day NM, et al. Body fat composition assessment using analytic morphomics predicts infectious complications after bowel resection in Crohn's disease. Inflamm Bowel Dis 2015;21:13061313.

14. Fehrman-Ekholm I, Elinder CG, Stenbeck M, Tyden G, Groth CG. Kidney donors live longer. Transplantation 1997;64:976-978.

15. Ramcharan T, Matas AJ. Long-term (20-37 years) follow-up of living kidney donors. Am J Transplant 2002;2:959-964.

16. MATLAB and Statistics Toolbox Release 2012b. Natick, MA: The MathWorks; 2012.

17. Wang SC, Holcombe SA, Derstine BA, et al. Reference analytic morphomics population (RAMP): a reference to measure occupant variability for crash injury analysis. In proceedings 2016 IRCOBI, International Research Council on the Biomechanics of Injury 2016; 582-591.

18. Zou H, Hastie T. Regularization and variable selection via the elastic net. J R Stat Soc Series B Stat Methodol 2005;67:301-320.

19. R Core Team. $R$ : a language and environment for statistical computing. Vienna, Austria: R Foundation for Statistical Computing; 2016

20. Nuttall FQ. Body mass index: obesity, BMI, and health: a critical review. Nutr Today 2015;50:117-128.

21. Bharadwaj S, Ginoya S, Tandon P, et al. Malnutrition: laboratory markers vs nutritional assessment. Gastroenterol Rep (Oxf) 2016;4:272-280.

22. Marcason W. Should albumin and prealbumin be used as indicators for malnutrition? J Acad Nutr Diet 2017;117:1144. 